\title{
Predictive role of NLR, SII, and PLR in COVID-19 patient mortality and disease severity
}

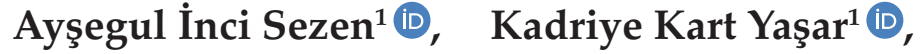
1 Health Sciences University, Bakırköy Dr. Sadi Konuk Education and Research Hospital, Department of Infectious Diseases and Clinical
Microbiology, Istanbul, Turkey

\begin{abstract}
Background: In this study, it was aimed to evaluate the factors affecting the prognosis and mortality of patients hospitalized for coronavirus disease 2019 (COVID-19).

Methods: Patients hospitalized with COVID-19 infection between March and November 2020 were examined retrospectively. The Systemic Immune-Inflammation Index (SII), platelet lymphocyte ratio (PLR), and neutrophil-lymphocyte ratio (NLR) values were evaluated for their effect on prognosis.

Results: Of the 1013 patients included in the study, 204 (20.1\%) had a severe infection. In the multivariate analysis, it was determined that the prognosis was significantly worse in patients who were $>65$ years of age, had a Charlson Comorbidity Index (CCI) score of $>2$, and had a high NLR rate. The C-reactive protein (CRP), PLR, SII values were detected as insignificant variables. Mortality was found to be statistically significant in patients with a CCI score of 2 or more and in patients with high CRP, NLR, PLR, and SII values at the time of admission $(\mathrm{p}<0.05)$ in the multivariate analysis.

Conclusions: It was found that the most important factor affecting the severity of the disease was advanced age and high comorbidities, and a high NLR value. The most important prognostic factors affecting mortality were high levels of comorbidities, and high NLR, PLR, SII, and CRP values.
\end{abstract}

Keywords: COVID-19, Mortality, Prognosis, Systemic immune-inflammation index, Neutrophil Lymphocyte Ratio 


\section{INTRODUCTION}

The new coronavirus disease 2019 (COVID-19), which is also known as severe acute respiratory syndrome 2 (SARS-CoV-2), has caused unpredictable high morbidity and mortality rates in many countries. The clinical spectrum of COVID-19 ranges from asymptomatic infection to multiorgan failure. In fact, the basic clinical picture is manifested by pneumonia and acute respiratory failure syndrome (ARDS) that develops in lung tissue and thromboembolic events in vital organs, such as the heart and brain (1).

Routine blood test results are generally used to diagnose inflammatory periods of infectious diseases. It is easy to perform inexpensive complete blood tests and provides blood cell types and morphological parameters such as White blood count, lymphocytes, neutrophils, monocytes, platelet count (PLT), and mean platelet volume (2). In addition, combined ratios of these parameters are also used as inflammation indexes and have been used to predict the diagnosis and progress of inflammatory/ infectious diseases (3). Neutrophil to lymphocyte ratio (NLR), platelet to lymphocyte ratio (PLR), and systemic inflammation response index (SII) can be helpful for the diagnosis and severity assessment of COVID-19 patients. The exaggerated and uncontrolled inflammatory response is one of the main reasons for COVID-19 disease severity. Systemic immune-inflammatory index (SII) is another parameter that reflects the immune and inflammatory status of the organism (4). Although there are many retrospective studies in the literature on COVID-19, there is still no clear consensus on prognostic factors affecting the disease (5). To date, there have been publications showing parameters such as leukocytosis, lymphopenia, increased lactate dehydrogenase, C-reactive protein (CRP), and increased interleukin 6 (IL-6) levels are factors that increase the mortality risk of the disease. However, studies on the effect of the Systemic Immune-Inflammation Index (SII) and neutrophil-lymphocyte ratios (NLR) on mortality and prognosis are still controversial (6).

This study aimed to evaluate the factors affecting the prognosis and mortality of patients hospitalized with COVID-19, as well as examine whether the SII is a useful tool in predicting 30-day mortality and identify other factors associated with higher mortality in COVID-19 patients.

\section{MATERIALS AND METHODS}

This study was conducted retrospectively with patients hospitalized with COVID-19 between March and November 2020, who were diagnosed with RT-PCR (realtime reverse transcriptase-polymerase chain reaction), or diagnosed with COVID-19 with radiological and clinical features. The epidemiological characteristics, clinical symptoms, and laboratory findings of the patients were taken from the patient files. Hemogram, biochemical tests, inflammation markers, and coagulation tests were conducted for each patient.

The criteria set by the World Health Organization (WHO) were used in the diagnosis of COVID-19 (7). The patients were diagnosed using the standard diagnostic method of studying RT-PCR in respiratory samples, such as nasopharyngeal swabs or sputum, as the test protocols defined by the WHO in the diagnosis of the disease (8). In patients with typical COVID-19 symptoms but negative test results for RT-PCR, a diagnosis of COVID-19 was made in the presence of diffuse ground-glass opacities or multiple viral pneumonic foci on chest computed tomography (CT) radiologically. Severity criteria were defined as set by WHO (9). In non-severe patients, their epidemiological history, fever or other respiratory symptoms, typical CT images of viral pneumonia, and positive RT-PCR results were available. Patients with severe infections had at least one of the following criteria: respiratory distress, a respiratory rate that was $\geq 30$, oxygen saturation (on room air) that was $\leq 93 \%, \mathrm{PaO}_{2} / \mathrm{FiO}_{2} \leq 300 \mathrm{mmHg}$ (9).

Systemic Immune-Inflammation Index (SII): Calculated with the formula (neutrophil $\times$ platelet count)/ lymphocyte. neutrophil-lymphocyte ratio (NLR): calculated as neutrophil/lymphocyte. The Charlson Comorbidity Index (CCI) was used for the comorbidities (10).

The study was approved by the institutional review board and was conducted in accordance with the principles of the Declaration of Helsinki. (Bakirkoy Dr. Sadi Konuk Eğitim ve Arastırma Hastanesi / 20-14-12 / 06-07-2020)

\section{Statistical Analysis}

Statistical analyses were conducted using IBM SPSS Statistics for Windows 22.0 (IBM Corp., Armonk, NY, USA). The numerical variables were expressed as the mean \pm standard deviation and median (min-max), and 
the categorical variables were expressed as numbers and percentages. Parametric test assumptions (normality and homogeneity of variances) were checked before comparing the groups in terms of numerical variables. The Mann-Whitney $U$ test was used to compare the continuous variables. Binary logistic regression analysis was performed among the statistically important factors. The optimal cut-off values of the continuous NLR, PLR, and SII were calculated by applying the receiver operating curve (ROC) analysis. The significance level was accepted as $\mathrm{p}<0.05$.

\section{RESULTS}

Of the 1013 patients included in the study, $552(54.5 \%)$ were male, $461(45.5 \%)$ were female, and their mean age was $54.31 \pm 16.20$ years (range: $16-95)$. While $734(72.5 \%)$ of the patients were $\leq 65$ years of age, 279 patients $(27.5 \%)$ were $>65$. While there were 288 patients $(28.4 \%)$ with a CCI score of 0 and 1 , there were $725(71.6 \%)$ patients with a CCI score of 2 or above. The median lymphocyte count of the patients was $1.23(1000 / \mu \mathrm{L})$, median neutrophil count was $3.93(1000 / \mu \mathrm{L})$, median platelet count was 195 $(1000 / \mu \mathrm{L})$, median CRP level was $44.87 \mathrm{mg} / \mathrm{dL}$. While the median NLR rate was 3.12, the median SII rate was 598.51. The median PLR of the patients was 157.89. The mean hospitalization period of the patients was $9.58 \pm 6.88$ days.

The disease category of 204 patients $(20.1 \%)$ was a severe infection. In the univariate analysis, the prognosis of 80 patients $(39.2 \%)$ who were $>65$ was significantly worse than those who were $\leq 65$ ( $\mathrm{p}<0.001) .170(83.3 \%)$ patients with a CCI of 2 and above had a significantly more severe prognosis than patients with a CCI of 0 to 1 . At the same time, the CRP, NLR, SII, and PLR values were significantly higher in the patients with poor prognoses $(p<0.05)$. Univariate analyses of the factors affecting the prognosis of the disease are shown in Table 1.

Table 1. Factors affecting the severity of the disease

\begin{tabular}{|c|c|c|c|c|}
\hline \multicolumn{2}{|c|}{ Variables } & \multirow{2}{*}{$\begin{array}{c}\text { Non-severe } \\
\mathrm{n}(\%)\end{array}$} & \multirow{2}{*}{$\begin{array}{l}\text { Severe } \\
\mathrm{n}(\%)\end{array}$} & \multirow{2}{*}{$\begin{array}{c}\text { Univariate } \\
\text { p-value }\end{array}$} \\
\hline & & & & \\
\hline \multirow[t]{2}{*}{ Age (Year) } & $<65$ & $610(75.4)$ & $124(60.8)$ & \multirow[t]{2}{*}{$<0.001$} \\
\hline & $>65$ & 199 (24.6) & $80(39.2)$ & \\
\hline \multirow[t]{2}{*}{ Gender } & Women & $443(54.8)$ & 109 (53.4) & \multirow[t]{2}{*}{0.734} \\
\hline & Men & 366 (45.2) & 95 (46.6) & \\
\hline \multirow[t]{2}{*}{ CCI } & $0-1$ & 254 (31.4) & 34 (16.7) & \multirow[t]{2}{*}{$<0.001$} \\
\hline & $\geq 2$ & $2.85 \pm 0.57$ & $2.34 \pm 0.37$ & \\
\hline $\mathrm{CRP}(\mathrm{mg} / \mathrm{L})$ & (Median) & 38.0 & 65.4 & $<0.001$ \\
\hline NLR & (Median) & 2.9 & 4.0 & $<0.001$ \\
\hline PLR & (Median) & 152.6 & 175.4 & 0.004 \\
\hline SII & (Median) & 576.9 & 744.1 & 0.009 \\
\hline
\end{tabular}

NLR: neutrophil to lymphocyte ratio, PLR: Platelet to lymphocyte ratio, SII: systemic immune inflammation index, CCI: Charlson Comorbidity Index Bold entries are statistically significant values. 
In the multivariate analysis, it was determined that the prognosis was significantly worse in the patients who were $>65$ years of age, and had a CCI score that was $>2$ and a high NLR rate. The CRP, PLR, SII values were detected as insignificant variables (Table 2).

Table 2. Multivariate analysis of factors affecting the severity of the disease

\begin{tabular}{|c|c|c|c|c|c|}
\hline \multicolumn{2}{|c|}{ Variables } & \multicolumn{2}{|c|}{$95 \% \mathrm{CI}$} & \multirow[t]{2}{*}{ HR } & \multirow{2}{*}{$\begin{array}{c}\text { Multivariate } \\
\text { p-value }\end{array}$} \\
\hline & & Lower & Upper & & \\
\hline Age & $>65$ & 1.1 & 2.2 & 1.5 & 0.011 \\
\hline CCI & $\geq 2$ & $199(24.6)$ & $80(39.2)$ & $0(3$ & $0(3$ \\
\hline CRP & & 0.9 & 1.0 & 1.0 & 0.514 \\
\hline NLR & & 1.0 & 1.1 & 1.0 & 0.001 \\
\hline PLR & & 1.0 & 1.0 & 1.0 & 0.151 \\
\hline SII & & 1.0 & 1.0 & 1.0 & 0.585 \\
\hline
\end{tabular}

NLR: neutrophil to lymphocyte ratio, PLR: Platelet to lymphocyte ratio, SII: systemic immune inflammation index, CCI: Charlson Comorbidity Index Bold entries are statistically significant values.

Due to the absence of a reference cut-off value on NLR mortality, the area under the curve (AUC) of the NLR value was determined as 0.614 in the ROC curve analysis
(Figure 1). The optimal NLR cut-off value was 3.38. The highest specificity and sensitivity were 0.585 and 0.603 for the NLR.

\section{Figure 1. The ROC curve analysis of NLR}

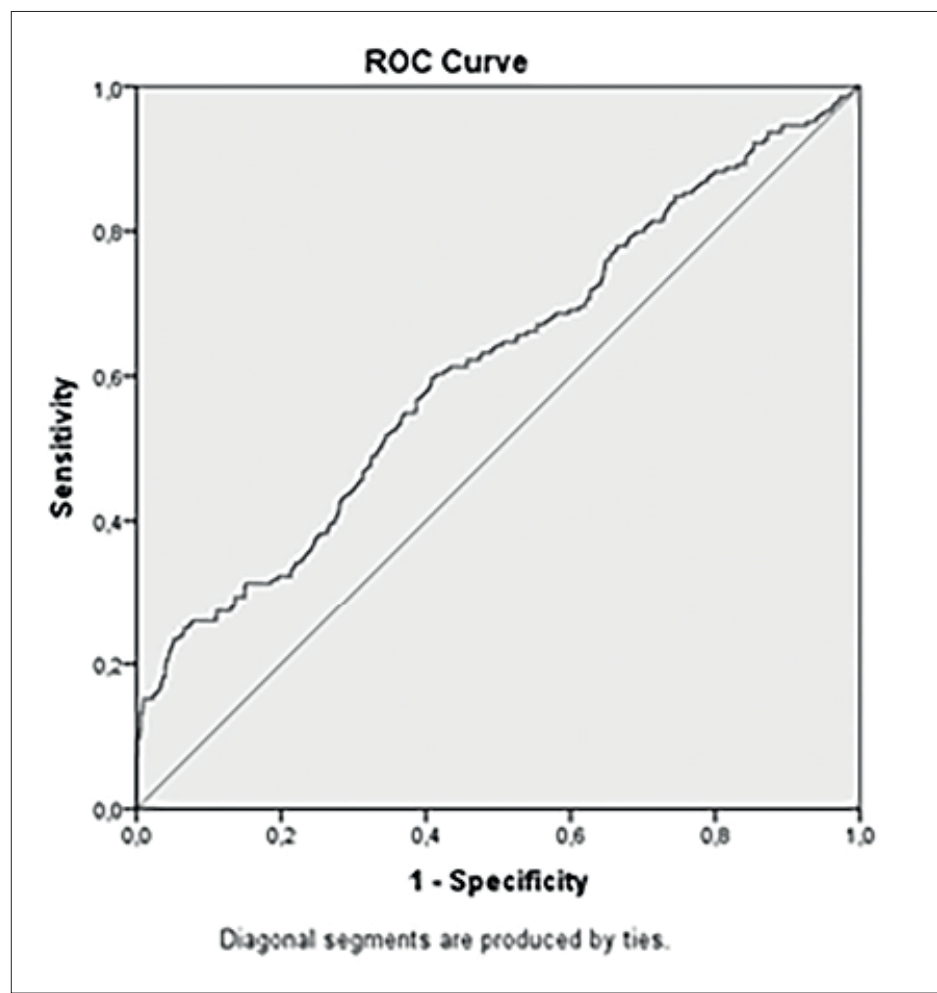


Mortality was observed in the first 30 days in 52 patients $(5.1 \%)$. In the univariate analysis, having a CCI score that was $>2$, and high CRP, NLR, PLR, and SII values were significant in terms of mortality. Mortality was observed in 45 patients $(86.5 \%)$ with a CCI index that was $>2$ $(p=0.014)$. The CRP, NLR, PLR, and SII values were higher in the patients with mortality $(\mathrm{p}<0.001)$ (Table 3$)$.

Table 3. Factors affecting the mortality of the disease

\begin{tabular}{|c|c|c|c|c|}
\hline \multicolumn{2}{|c|}{ Variables } & \multirow{2}{*}{$\begin{array}{l}\text { No Mortality } \\
\mathrm{n}(\%)\end{array}$} & \multirow{2}{*}{$\begin{array}{c}\text { With Mortality } \\
\mathrm{n}(\%)\end{array}$} & \multirow{2}{*}{$\begin{array}{c}\text { Univariate } \\
\text { p-value }\end{array}$} \\
\hline & & & & \\
\hline \multirow[t]{2}{*}{ Age (Year) } & $<65$ & $694(72.2)$ & $40(76.9)$ & \multirow[t]{2}{*}{0.459} \\
\hline & $>65$ & $267(27.8)$ & $12(23.1)$ & \\
\hline \multirow[t]{2}{*}{ Gender } & Men & $524(54.5)$ & $28(53.8)$ & \multirow[t]{2}{*}{0.924} \\
\hline & Women & $437(45.5)$ & $24(46.2)$ & \\
\hline \multirow[t]{2}{*}{$\mathrm{CCI}$} & $0-1$ & $281(29.2)$ & 7 (13.5) & \multirow[t]{2}{*}{0.014} \\
\hline & $\geq 2$ & $2.85 \pm 0.57$ & $2.34 \pm 0.37$ & \\
\hline $\mathrm{CRP}(\mathrm{mg} / \mathrm{L})$ & (Median) & 41 & 126 & $<0.001$ \\
\hline NLR & (Median) & 3.0 & 6.64 & $<0.001$ \\
\hline PLR & (Median) & 155.2 & 226.1 & $<0.001$ \\
\hline SII & (Median) & 578.4 & 1437.6 & $<0.001$ \\
\hline
\end{tabular}

NLR: neutrophil to lymphocyte ratio, PLR: Platelet to lymphocyte ratio, SII: systemic immune inflammation index, CCI: Charlson Comorbidity Index Bold entries are statistically significant values.

Mortality was found to be statistically significant in patients with a CCI score that was $>2$ and in patients with high CRP, NLR, PLR, and SII values at the time of admission $(\mathrm{p}<0.05)$ in the multivariate analysis affecting mortality (Table 4 ).

Table 4. Multivariate analysis of the factors affecting the mortality of the disease

\begin{tabular}{|c|c|c|c|c|c|}
\hline \multicolumn{2}{|c|}{ Variables } & \multicolumn{2}{|c|}{$95 \%$ CI } & \multirow[t]{2}{*}{ HR } & \multirow{2}{*}{$\begin{array}{c}\text { Multivariate } \\
\text { p-value }\end{array}$} \\
\hline & & Lower & Upper & & \\
\hline $\mathrm{CCI}$ & $\geq 2$ & 1.1 & 2.2 & 1.5 & 0.011 \\
\hline CRP & & 1.0 & 1.0 & 1.0 & 0.001 \\
\hline NLR & & 1.0 & 1.1 & 1.0 & 0.032 \\
\hline PLR & & 1.0 & 1.0 & 1.0 & 0.001 \\
\hline SII & & 0.9 & 1 & 1.0 & 0.038 \\
\hline
\end{tabular}

NLR: neutrophil to lymphocyte ratio, PLR: Platelet to lymphocyte ratio, SII: systemic immune inflammation index, CCI: Charlson Comorbidity Index Bold entries are statistically significant values. 
There is no reference cut-off value for the NLR, CRP, SII, and PLR on mortality. Therefore, in the ROC curve analysis, the AUC of the CRP, NLR, SII, PLR values were determined as $0.734,0.761,0.708$, and 0.723 . The optimal cut-off values of the CRP, NLR, SII, and PLR were 9.02, 4.15, 909.5, and 181.5. The highest specificity and sensitivity were 0.981 and $0.837,0.750$ and $0.664,0.692$ and 0.683 , and 0.673 and 0.626 , respectively, for the CRP, NLR, SII, PLR.

\section{DISCUSSION}

The NLR, PLR, SII are ratios of hematological cells that regulate inflammatory response during infection and injury. The importance of these ratios has been researched in a wide variety of diseases, including malignancies (11,12). In addition to taking advantage of the relationship between tests such as the PLR, NLR, SII, CRP, and inflammatory conditions, the main benefit of these tests is that they are easily measurable and done more frequently and routinely than other tests. Most importantly, they are cost-effective, and they will not affect the already overburdened healthcare system. In light of this information, during the epidemic that has caused millions of deaths, any cost-effective method that could provide a prediction for the severity of the spectrum will significantly guide clinicians during treatment and follow-up.

The NLR values in the studies conducted by Liu et al. (13) and Fu et al. (14) in China were found to be an independent predictor factor in COVID-19 patients. In the study conducted by Kalabin et al. (15), the NLR and PLR values were significantly increased in COVID-19 patients, although these inflammatory markers were not associated with disease severity. In the current study, the NLR value was significantly increased with the disease severity, while the PLR value was not significant. In the study of Merad et al. (16), it was found that COVID-19 triggered hyper inflammation, and similar findings were found in the current study. While many inflammatory markers, such as CRP, erythrocyte sedimentation rate, lactate dehydrogenase, ferritin, and procalcitonin are frequently measured, the NLR and PLR values, which are particularly cost-effective in COVID-19 patients, can also be easily calculated. In the present study, patients with severe COVID-19 had higher NLR values when compared to those without the severe disease. In a meta-analysis performed by Chan et al. (17), the NLR values were found to be significantly higher in severe
COVID-19. Patients with severe COVID-19 presented with increased leukocytosis, neutrophilia, lymphopenia, and thrombocytopenia when compared to those with non-severe disease (18). These patients are more likely to develop ARDS and may require intensive care unit (ICU) level care (19). Different mechanisms of lymphopenia in COVID-19 patients may be that the virus infects T-cells via ACE2 receptors (20). Decreased CD4 + and CD8 + T lymphocyte levels are associated with the severity of the disease, which can lead to an increased NLR (21). In the present study, the NLR value was significant when the factors affecting both the severity of the disease $(p<0.001)$ and mortality $(\mathrm{p}<0.05)$ were considered.

Platelets are immune cells that play an important role in the human body. The meta-analysis investigating the relationship between thrombocytopenia and COVID-19 severity showed that the platelet count tripled the risk of serious illness and death in COVID-19 patients (22). According to another study, increased PLR levels observed in the follow-up of symptomatic and asymptomatic patients were found to be associated with the severity of the disease and the length of hospital stay (4). There have been publications stating that a new indicator can be obtained in the cytokine storm monitoring of patients with high PLR levels (23). In another study conducted in the USA, it was reported that the increase in the PLR value had no effect on the severity of the disease (15). In the current study, statistically, the increase in the PLR value was not significant in the disease severity, but it was significant in mortality.

As a result of our study, we found statistically significant results between the increase in the CRP levels and increased mortality $(\mathrm{p}<0.001)$. Zeng et al. (24) mentioned that inflammatory markers were important factors of the COVID-19 severity. In their meta-analysis, they found that non-severe group had lower levels for CRP (41.78 $\mathrm{mg} / 1,95 \% \mathrm{CI}=[31.1-52.4], \mathrm{p}<0.001)$ compared with those in the severe group. Hariyanto et al. (25) had written a systematic review and meta-analysis supporting data that elevated CRP levels can be used for predicting severe outcomes in COVID-19. In the data obtained by Ullah et al. (26), the relationship between higher CRP levels and COVID-19 were found to be correlated with sepsis, ICU admission, and increased mortality.

In a retrospective study conducted in Italy, the SII was compared with other commonly used blood cell count- 
based inflammation indices, such as the NLR and PLR in COVID-19 patients, and was found to be the most significant survival factor. In the present study, the SII was found to be significant among the factors affecting mortality $(p<0.05)$. When the CCI was first invented, it was used to predict the risk of death within one year of hospital stay. The scores are based on a series of comorbidities, which are each given a number from 1 to 6 , depending on the severity of the morbidity (27). It is a well-validated, simple, and easy-to-apply index for evaluating the prognosis and survival of patients. During the current pandemic, the severity and mortality of COVID-19 has often been estimated using age, gender, and the presence of comorbidities, such as diabetes, cardiovascular, cerebrovascular, and respiratory diseases. The mortality and risk factors in COVID-19 patients can be evaluated by calculating the CCI score, age, and accompanying comorbidities together. In a study conducted by Imam et al. (28) in the USA, advanced age and multiple comorbidities were specified as independent mortality risk factors in patients with COVID-19.

In the present study, a CCI score that was $\geq 2$ significantly affected both the severity of the disease and mortality $(p<0.05)$. Age was found to be a significant factor affecting the severity of the disease, independent of the CCI score $(\mathrm{p}<0.001)$. As the pandemic continues worldwide, it is essential to understand the clinical characteristics of patients and the risk factors of worse outcomes in COVID-19 to be able to plan comprehensive treatment and appropriately allocate valuable resources (29).

Due to the nature of this study, it is retrospective and single-centered, which creates a major bias. The inflammatory factors, which were found to be significant regarding mortality and morbidity, constituted a bias in this case, because these inflammatory factors also increase in all infectious diseases.

As a result of the current study, it was found that the most important factor affecting the severity of the disease was advanced age and high comorbidities, and a high NLR value. The most important prognostic factors affecting mortality were high levels of comorbidities, and high NLR, PLR, SII, and CRP values. These analyses can be useful in evaluating COVID-19 patients, as they are simple, cost-effective, and rapid laboratory diagnostic tests.

\section{Declarations}

The authors received no financial support for the research and/or authorship of this article. There is no conflict of interest.

The study was approved by the institutional review board and was conducted in accordance with the principles of the Declaration of Helsinki. (Bakirkoy Dr. Sadi Konuk Eğitim ve Arastırma Hastanesi/ 20-1412/06-07-2020)

\section{REFERENCES}

1. Ma X, Ng M, Xu S, Xu Z, Qiu H, Liu Y, et al. Development and validation of prognosis model of mortality risk in patients with COVID-19. Epidemiol Infect. 2020;148.

2. Usul E, Şan İ, Bekgöz B, Şahin A. Role of hematological parameters in COVID-19 patients in the emergency room. Biomark Med. 2020;14(13):1207-15.

3. Peng J, Qi D, Yuan G, Deng X, Mei Y, Feng L, et al. Diagnostic value of peripheral hematologic markers for coronavirus disease 2019 (COVID-19): A multicenter, cross-sectional study. J Clin Lab Anal. 2020;34(10):e23475.

4. Yang AP, Liu JP, Tao WQ, Li HM. The diagnostic and predictive role of NLR, d-NLR and PLR in COVID-19 patients. Int Immunopharmacol. 2020;84:106504.

5. Pourbagheri-Sigaroodi A, Bashash D, Fateh F, Abolghasemi H. Laboratory findings in COVID-19 diagnosis and prognosis. Clin Chim Acta. 2020;510:475

6. Fois AG, Paliogiannis P, Scano V, Cau S, Babudieri S, Perra R, et al. The systemic inflammation index on admission predicts in-hospital mortality in COVID-19 patients. Molecules. 2020;25(23):5725.

7. World Health Organization. Clinical management of severe acute respiratory infection when Middle East respiratory syndrome coronavirus (MERS-CoV) infection is suspected: interim guidance. 2019. Available at: https://apps.who.int/iris/ handle/10665/178529 Accessed February 11, 2020.

8. World Health Organization. Laboratory testing for 2019 novel coronavirus (2019-nCoV) in suspected human cases: interim guidance. 2020. Available at: https://www.who.int/ publications/i/item/10665-331501 Accessed January 14, 2020.

9. World Health Organization. Clinical management of severe acute respiratory infection when novel coronavirus (2019-nCoV) infection is suspected. 2019. https:/ / www.who.int/csr/ disease/ coronavirus_infections/InterimGuidance_ClinicalManage ment NovelCoronavirus_11Feb13u.pdf?ua $=1 \& u a=1$. Accessed February 11, 2020.

10. Beddhu S, Bruns FJ, Saul M, Seddon P, Zeidel ML. A simple comorbidity scale predicts clinical outcomes and costs in dialysis patients. Am J Med. Elsevier; 2000;108:609-13.

11. Duan J, Pan L, Yang M. Preoperative elevated neutrophil-tolymphocyte ratio (NLR) and derived NLR are associated with poor prognosis in patients with breast cancer: A meta-analysis. Medicine (Baltimore). 2018;97(49):e13340. 
12. Diem S, Schmid S, Krapf M, Flatz L, Born D, Jochum W, et al. Neutrophil-to-Lymphocyte ratio (NLR) and Platelet-toLymphocyte ratio (PLR) as prognostic markers in patients with non-small cell lung cancer (NSCLC) treated with nivolumab. Lung Cancer. 2017;111:176-181.

13. Liu Y, Du X, Chen J, Jin Y, Peng L, Wang HHX, et al. Neutrophilto-lymphocyte ratio as an independent risk factor for mortality in hospitalized patients with COVID-19. J Infect. 2020;81(1):e6-e12.

14. Fu J, Kong J, Wang W, Wu M, Yao L, Wang Z, et al. The clinical implication of dynamic neutrophil to lymphocyte ratio and D-dimer in COVID-19: A retrospective study in Suzhou China. Thromb Res. 2020;192:3-8.

15. Kalabin A, Mani VRK, Valdivieso SC, Donaldson B. Role of neutrophil-to-lymphocyte, lymphocyte-to-monocyte, and platelet-to-lymphocyte ratios as predictors of disease severity in COVID-19 patients. Le Infez Med. 2021;29:46-53.

16. Merad M, Martin JC. Pathological inflammation in patients with COVID-19: a key role for monocytes and macrophages. Nat Rev Immunol. 2020;20(6):355-362.

17. Chan AS, Rout A. Use of neutrophil-to-lymphocyte and plateletto-lymphocyte ratios in COVID-19. J Clin Med Res. 2020;12:448.

18. Henry BM, De Oliveira MHS, Benoit S, Plebani M, Lippi G. Hematologic, biochemical and immune biomarker abnormalities associated with severe illness and mortality in coronavirus disease 2019 (COVID-19): a meta-analysis. Clin Chem Lab Med. 2020;58:1021-8.

19. Wu C, Chen X, Cai Y, Zhou X, Xu S, Huang H, et al. Risk factors associated with acute respiratory distress syndrome and death in patients with coronavirus disease 2019 pneumonia in Wuhan, China. JAMA Intern Med. 2020;180:934-43.

20. Wang K, Chen W, Zhang Z, Deng Y, Lian JQ, Du P, et al. CD147spike protein is a novel route for SARS-CoV-2 infection to host cells. Signal Transduct Target Ther. 2020;5(1):283.

21. Ye W, Chen G, Li X, Lan X, Ji C, Hou M, et al. Dynamic changes of D-dimer and neutrophil-lymphocyte count ratio as prognostic biomarkers in COVID-19. Respir Res. 2020;21:1-7.

22. Liu YP, Li GM, He J, Liu Y, Li M, Zhang R, et al. Combined use of the neutrophil-to-lymphocyte ratio and CRP to predict 7-day disease severity in 84 hospitalized patients with COVID-19 pneumonia: a retrospective cohort study. Ann Transl Med. 2020;8(10):635

23. Qu R, Ling Y, Zhang Y, Wei L, Chen X, Li X, et al. Platelet-tolymphocyte ratio is associated with prognosis in patients with coronavirus disease-19. J Med Virol. 2020;92:1533-41.

24. Zeng F, Huang Y, Guo Y, Yin M, Chen X, Xiao L, et al. Association of inflammatory markers with the severity of COVID-19: A metaanalysis. Int J Infect Dis. 2020;96:467-474.

25. Hariyanto TI, Japar KV, Kwenandar F, Damay V, Siregar JI, Lugito $\mathrm{NPH}$, et al. Inflammatory and hematologic markers as predictors of severe outcomes in COVID-19 infection: A systematic review and meta-analysis. Am J Emerg Med. 2021;41:110-119.

26. Ullah W, Thalambedu N, Haq S, Saeed R, Khanal S, Tariq S, et al. Predictability of CRP and D-Dimer levels for in-hospital outcomes and mortality of COVID-19. J community Hosp Intern Med Perspect. 2020;10:402-8.

27. Austin SR, Wong YN, Uzzo RG, Beck JR, Egleston BL. Why Summary Comorbidity Measures Such As the Charlson Comorbidity Index and Elixhauser Score Work. Med Care. 2015;53(9):e65-72.
28. Imam Z, Odish F, Gill I, O'Connor D, Armstrong J, Vanood A, et al. Older age and comorbidity are independent mortality predictors in a large cohort of 1305 COVID-19 patients in Michigan, United States. J Intern Med. 2020;288:469-76.

29. Kuswardhani RAT, Henrina J, Pranata R, Lim MA, Lawrensia S, Suastika K. Charlson comorbidity index and a composite of poor outcomes in COVID-19 patients: A systematic review and metaanalysis. Diabetes Metab Syndr Clin Res Rev. Elsevier; 2020; 